\title{
Study on a Novel High-Efficiency Bridgeless PFC Converter
}

\author{
Cao Taiqiang, ${ }^{1}$ Chen Zhangyong, ${ }^{2}$ Wang Jun, ${ }^{1}$ Sun Zhang, ${ }^{1}$ Luo Qian, ${ }^{3}$ and Zhang Fei ${ }^{2}$ \\ ${ }^{1}$ School of Electric Information, Xihua University, Chengdu 610039, China \\ ${ }^{2}$ School of Electrical Engineering, Southwest Jiaotong University, Chengdu 610031, China \\ ${ }^{3}$ Information Technology Branch, The Second Research Institute of General Administration of Civil Aviation of China, \\ Chengdu 610042, China
}

Correspondence should be addressed to Cao Taiqiang; ctq815@163.com

Received 24 January 2014; Accepted 18 June 2014; Published 4 August 2014

Academic Editor: Gongnan Xie

Copyright (C) 2014 Cao Taiqiang et al. This is an open access article distributed under the Creative Commons Attribution License, which permits unrestricted use, distribution, and reproduction in any medium, provided the original work is properly cited.

In order to implement a high-efficiency bridgeless power factor correction converter, a new topology and operation principles of continuous conduction mode (CCM) and DC steady-state character of the converter are analyzed, which show that the converter not only has bipolar-gain characteristic but also has the same characteristic as the traditional Boost converter, while the voltage transfer ratio is not related with the resonant branch parameters and switching frequency. Based on the above topology, a novel bridgeless Bipolar-Gain Pseudo-Boost PFC converter is proposed. With this converter, the diode rectifier bridge of traditional ACDC converter is eliminated, and zero-current switching of fast recovery diode is achieved. Thus, the efficiency is improved. Next, we also propose the one-cycle control policy of this converter. Finally, experiments are provided to verify the accuracy and feasibility of the proposed converter.

\section{Introduction}

A great number of harmonic currents, which are caused by nonlinear loads connected to grid, are main pollution sources of power system and important factors in the safe operation of the grid. In order to reduce the harmonic pollution, the active power filter (APF) and power factor correction (PFC) are required, which are applied to control the harmonic and reactive current generated from rectifier loads. PFC converter can realize the AC input current control and DC output voltage control at the same frequency and phase with AC voltage, and thus it is widely used in server power [1-8], charger, UPS, and so on. Traditional PFC converter includes a front-end bridge rectifier, which increases conduction loss and greatly affects the efficiency of the PFC converter. Moreover, more serious loss is generated under low input voltage and light load particularly [5,9-13].

In order to improve efficiency of the traditional PFC converter, an effective method named as bridgeless PFC technology without front-end bridge rectifier is proposed $[3,14,15]$. Then the bridgeless PFC converter is proposed in [1-18], which uses two switches instead of the uncontrolled diodes. When AC input voltage is in positive half-cycle, the front bridge leg of the converter operations, while AC input voltage is in negative half-cycle, the rear bridge leg operations. The papers report that the bridgeless PFC converter adopts the same two DC-DC converters to implement the power conversion for positive and negative half-cycle of AC input voltage, respectively. Another bridgeless PFC converter makes the input voltage greater than zero at first and then obtains steady output voltage through the DCDC converter. All of these bridgeless PFC converters have a higher cost, require complex topology, and may lead to serious electromagnetic inference (EMI) [3, 13-18].

In this paper, we research a novel Pseudo-Boost converter, which has bipolar-gain characteristic; that is, no matter if the input voltage is positive or negative, the output voltage is always positive, and the converter also has the same characteristic as the traditional Boost converter. Although the converter has a resonant branch, the difference from the traditional resonant converter is that the voltage transfer ratio is not relevant to the resonant branch parameters and switching frequency; it only depends on the switching duty cycle. Based on the Bipolar-Gain Pseudo-Boost converter, 


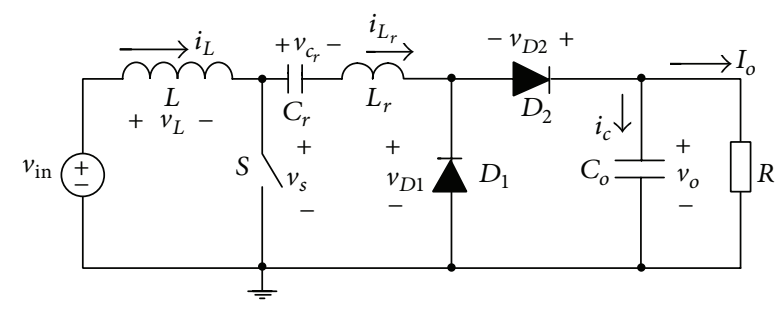

Figure 1: The topology of Bipolar-Gain Pseudo-Boost converter.

a bridgeless Bipolar-Gain Pseudo-Boost PFC converter in continuous conduction mode (CCM) is investigated. The converter adopts one bidirectional controllable switch and two fast-recovery diodes instead of bridge rectifier, and the zero-current switching of the fast-recovery diodes is achieved. Therefore, the efficiency of the converter is improved. Meanwhile, the one-cycle control technology is adopted to control the Bipolar-Gain Pseudo-Boost PFC converter.

\section{Operation Principle of Bipolar-Gain Pseudo-Boost Converter}

The topology of the Bipolar-Gain Pseudo-Boost converter consists of switch $S$, two recovery diodes $D_{1}$ and $D_{2}$, resonant capacitor $C_{r}$, and resonant inductor $L_{r}$, as in Figure 1. When the input voltage is positive or negative, the output voltage of traditional Boost converter is the corresponding polarity. Compared with the traditional one, the output voltage of Bipolar-Gain Pseudo-Boost converter is just positive; that is, the Bipolar-Gain Pseudo-Boost has bipolar-gain characteristic. To simplify the analysis of the operation of the BipolarGain Pseudo-Boost converter, the following assumptions are made.

(a) All of the switches, diodes, inductors, and capacitors are ideal.

(b) Output capacitor $C_{o}$ is large enough to make the output voltage keep constant in a switching cycle.

(c) Resonant inductor $L_{r}$ and resonant capacitor $C_{r}$ are much smaller than the inductor $L$ and output capacitor $C_{o}$, respectively.

(d) The converter operates in CCM.

2.1. Positive Input Voltage. The operation modes of BipolarGain Pseudo-Boost converter operate in CCM with positive input voltage (Figure 2). The corresponding key waveforms of the converter are shown in Figure 3. At the beginning of each switching cycle, the resonant inductor current $i_{L_{r}}$ is zero and the initial value of resonant capacitor voltage $v_{c_{r}}$ is $\Delta v_{c_{r}}$, where $v_{c}>\Delta v_{c_{r}}>0$ exists.

(1) Mode $1\left[t_{0} \sim t_{1}\right]$ : as shown in Figures 2(a) and 3, at $t=t_{0}$, the switch $S$ is turned on. The voltage across the inductor $L$ is equal to input voltage $v_{\text {in }}$, which makes inductor current increase linearly. The resonant capacitor voltage $v_{c_{r}}$ makes diode $D_{1}$ turn on, and the reverse voltage across $D_{2}$ makes it turn off. Resonant capacitor voltage $v_{c_{r}}$ and resonant inductor current $i_{L_{r}}$ are given as

$$
\begin{gathered}
v_{c_{r}}=\Delta v_{c_{r}} \cos \omega_{r}\left(t-t_{0}\right), \\
i_{L_{r}}=-\frac{\Delta v_{c_{r}}}{Z_{n}} \sin \omega_{r}\left(t-t_{0}\right),
\end{gathered}
$$

where $Z_{n}=\sqrt{L_{r} / C_{r}}, \omega_{r}=1 / \sqrt{L_{r} C_{r}}$.

(2) Mode $2\left[t_{1} \sim t_{2}\right]$ : as shown in Figures $2(\mathrm{~b})$ and 3 , at $t=$ $t_{1}=t_{0}+T_{r} / 2$, resonant inductor current $i_{L_{r}}$ is equal to zero and diode $D_{1}$ gets zero-current turn-off, where $T_{r}=2 \pi / \omega_{r}$ is the resonant period. At this moment, the resonant capacitor is equal to $-\Delta v_{c_{r}}$. Due to $v_{c}>\Delta v_{c_{r}}$, the diode $D_{2}$ is still turned off with reverse voltage $\Delta v_{c_{r}}-v_{c}$. The voltage across diode $D_{1}$ is equal to $v_{D 1}=-v_{c_{r}}=\Delta v_{c_{r}}$, while the voltage across diode $D_{2}$ is equal to $v_{D 2}=v_{c}-\Delta v_{c_{r}}$.

(3) Mode $3\left[t_{2} \sim t_{3}\right]$ : as shown in Figures 2(c) and 3, at $t=t_{2}$, the switch $S$ is turned off. Because the inductor current $i_{L}$ does not change suddenly, the diode $D_{2}$ is turned on to provide the path for the inductor current $i_{L}$. The diode $D_{1}$ is reversely biased and the voltage across it is equal to $-v_{c}$, and the inductor $L$ is then discharged and capacitor $C_{o}$ is charged. As $L_{r} \ll L, L_{r}$ can be ignored. So during $t_{2} \sim t_{3}$, the resonant capacitor voltage can be expressed as

$$
v_{c r}=-\Delta v_{c_{r}}+\frac{i_{L}}{C_{r}}\left(t-t_{2}\right) .
$$

When the resonant capacitor voltage rises to $\Delta v_{c_{r}}$, the converter begins to enter the next switch cycle.

2.2. Negative Input Voltage. Figure 4 shows the operation modes of Bipolar-Gain Pseudo-Boost converter operated in CCM with negative input voltage. The corresponding key waveforms of the converter are shown in Figure 5. At the beginning of each switching cycle, the resonant inductor current $i_{L_{r}}$ is zero and the initial value of resonant capacitor voltage $v_{c_{r}}=-v_{c}-\Delta v_{c_{r}}$, where $v_{c}>\Delta v_{c_{r}}>0$.

(1) Mode $4\left[t_{4} \sim t_{5}\right]$ : as shown in Figures $4(\mathrm{a})$ and 5, at $t=t_{4}$, the switch $S$ is turned on. The voltage across the inductor $L$ is equal to input voltage $-v_{\text {in }}$, which makes inductor current decrease linearly. The diode $D_{2}$ is turned on by the opposite voltage $\Delta v_{c_{r}}$, and the diode $D_{1}$ is reversely biased. The resonant branch begins to resonate; the resonant capacitor voltage $v_{c_{r}}$ and resonant inductor current $i_{L_{r}}$ are given as

$$
\begin{gathered}
v_{c r}=-v_{c}-\Delta v_{c_{r}} \cos \omega_{r}\left(t-t_{4}\right), \\
i_{L r}=\frac{\Delta v_{c_{r}}}{Z_{n}} \sin \omega_{r}\left(t-t_{4}\right),
\end{gathered}
$$

where $Z_{n}=\sqrt{L_{r} / C_{r}}, \omega_{r}=1 / \sqrt{L_{r} C_{r}}$.

(2) Mode $5\left[t_{5} \sim t_{6}\right]$ : as shown in Figures 4(b) and 5, at $t=t_{5}$, the resonant inductor current $i_{L_{r}}$ is equal to zero and diode $D_{2}$ gets zero-current turn-off, where $t_{5}-t_{4}=T_{r} / 2$ and $T_{r}=2 \pi / \omega_{r}$ is the resonant period. The resonant capacitor is equal to $-v_{c}+\Delta v_{c_{r}}$, the diode $D_{1}$ is still reversely biased, and 


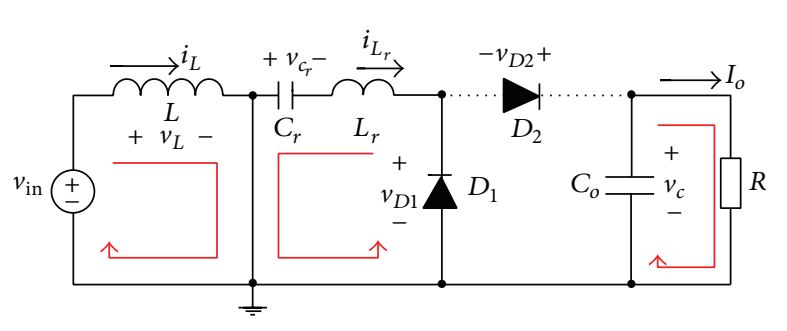

(a) Mode 1

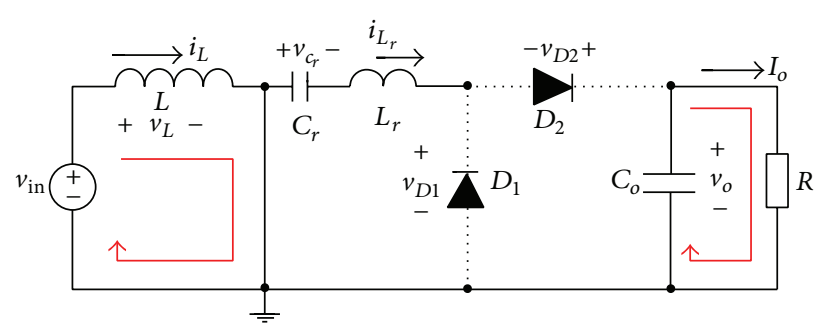

(b) Mode 2

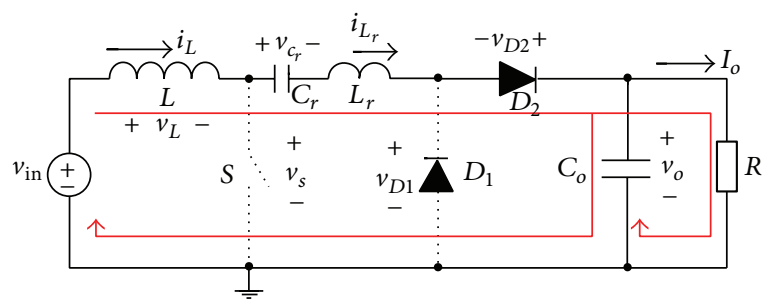

(c) Mode 3

FIGURE 2: Equivalent circuit of operation mode of the converter with positive input voltage.

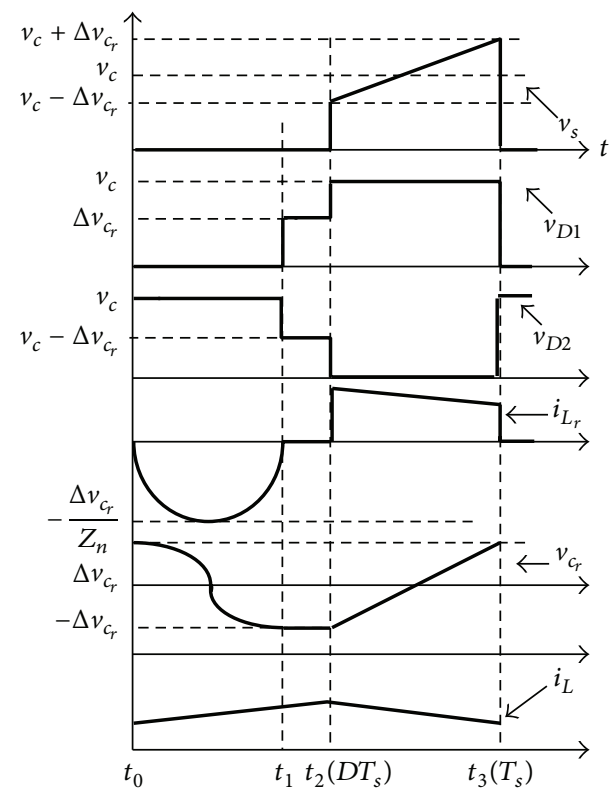

FIGURE 3: The key waveform of the converter with positive input voltage.

the voltage across it is equal to $v_{D 1}=-v_{c_{r}}=v_{c}-\Delta v_{c_{r}}$. The voltage across diode $D_{2}$ is equal to $\Delta v_{c_{r}}$.

(3) Mode $6\left[t_{6} \sim t_{7}\right]$ : as shown in Figures 4(c) and 5, at $t=t_{6}$, the switch $S$ is turned off. Because the inductor current $i_{L}$ does not change suddenly, the diode $D_{1}$ is turned on to provide the path for the inductor current $i_{L}$. The diode $D_{2}$ is reversely biased, and the inductor $L$ is then discharged and capacitor $C_{o}$ is charged. During $t_{6} \sim t_{7}$, the resonant capacitor voltage can be given as

$$
v_{c_{r}}=-v_{c}+\Delta v_{c_{r}}-\frac{i_{L}}{C_{r}}\left(t-t_{6}\right)
$$

When the resonant capacitor voltage decreases to $-v_{c}-$ $\Delta v_{c_{r}}$, the converter begins to enter the next switch cycle.

\section{The Steady-State Analysis of Bipolar-Gain Pseudo-Boost Converter}

There are four state variables in Bipolar-Gain Pseudo-Boost converter. One is the energy storage state variable $\left(v_{c}, i_{L}\right)$, in which the natural frequency is much lower than the switching frequency. The other one is the resonant state variables $\left(v_{c_{r}}, i_{L_{r}}\right)$, in which the natural frequency is close to the switching frequency. According to the generalized state space averaging (GSSA) approach, the GSSA equation of the converter can be established, which chooses $v_{c}, i_{L}$ as state variables. Based on the GSSA equation, the steady-state characteristic can be analyzed.

3.1. Positive Input Voltage. For positive input voltage, from Figures 2(a) and 2(b), when the switch $S$ is turned on, the state differential equations are

$$
\begin{gathered}
L \frac{\mathrm{d} i_{L}}{\mathrm{~d} t}=v_{\mathrm{in}}, \\
C_{o} \frac{\mathrm{d} v_{c}}{\mathrm{~d} t}=-\frac{v_{c}}{R} .
\end{gathered}
$$

From Figure 2(c), when the switch $S$ is turned off, the corresponding state differential equations are

$$
\begin{aligned}
\left(L+L_{r}\right) \frac{\mathrm{d} i_{L}}{\mathrm{~d} t} & =v_{\text {in }}-v_{c}-v_{c_{r}}, \\
C_{o} \frac{\mathrm{d} v_{c}}{\mathrm{~d} t} & =i_{L}-\frac{v_{c}}{R} .
\end{aligned}
$$




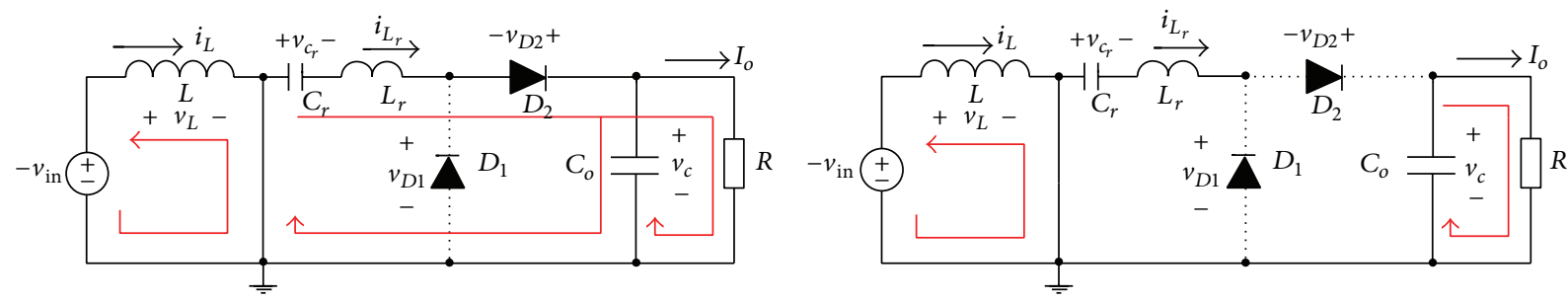

(a) Mode 4

(b) Mode 5

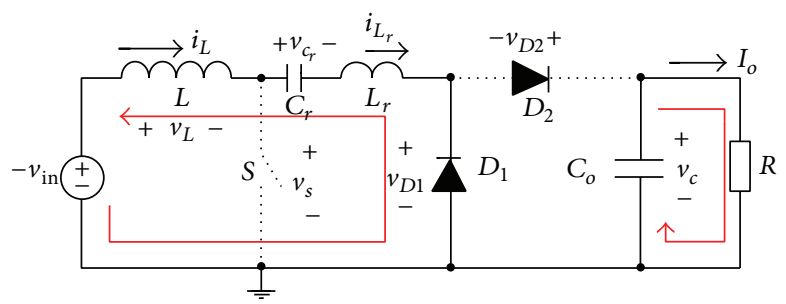

(c) Mode 6

FIGURE 4: Equivalent circuit of operation mode of the converter with negative input voltage.

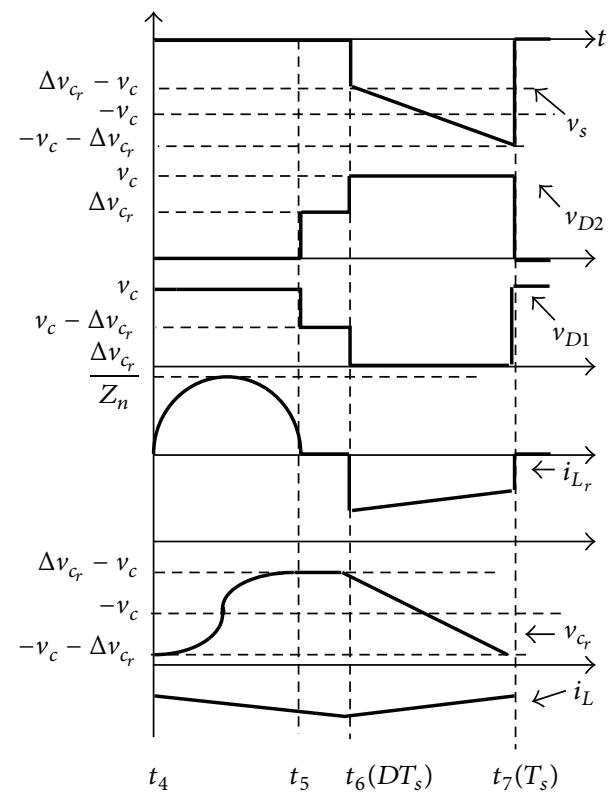

FIGURE 5: The key waveform of the converter with negative input voltage.

The above analysis shows that the resonant capacitor voltage satisfies (2) when the switch $S$ is turned off. From Figure 3, at the end of switching cycle, the resonant capacitor voltage is equal to $\Delta v_{c_{r}}$; meanwhile, the integration of the resonant capacitor voltage is zero.

The matrix representation of the state variables is $\mathbf{x}=$ $\left[\begin{array}{ll}i_{L} & v_{c}\end{array}\right]^{T}$; according to (5) and (6), the state space equation is

$$
\dot{\mathbf{x}}=A_{i} \mathbf{x}+B_{i} \mathbf{u}, \quad i=1,2
$$

where

$$
\begin{array}{cc}
A_{1}=\left[\begin{array}{cc}
0 & 0 \\
0 & -\frac{1}{R C_{o}}
\end{array}\right], & B_{1}=\left[\begin{array}{c}
\frac{v_{\text {in }}}{L} \\
0
\end{array}\right], \\
A_{2}=\left[\begin{array}{cc}
0 & -\frac{1}{L+L_{r}} \\
\frac{1}{C_{o}} & -\frac{1}{R C_{o}}
\end{array}\right], & B_{2}=\left[\begin{array}{c}
\frac{v_{\text {in }}-v_{c_{r}}}{L+L_{r}} \\
0
\end{array}\right] .
\end{array}
$$

According to GSSA approach, for positive input voltage, the GSSA equation of the Bipolar-Gain Pseudo-Boost converter can be obtained as

$$
\frac{\mathrm{d}}{\mathrm{d} t}\left[\begin{array}{l}
i_{L} \\
v_{c}
\end{array}\right]=\left[\begin{array}{cc}
0 & -\frac{(1-d)}{L+L_{r}} \\
\frac{(1-d)}{C_{o}} & -\frac{1}{R C_{o}}
\end{array}\right]\left[\begin{array}{c}
i_{L} \\
v_{c}
\end{array}\right]+\left[\begin{array}{c}
\frac{v_{\text {in }}}{L} \\
0
\end{array}\right],
$$

where $d$ is the transient state switching duty cycle.

The equation $(\mathrm{d} / \mathrm{d} t)\left[\begin{array}{l}i_{L} \\ v_{c}\end{array}\right]=0$ is founded at steady state because $L \gg L_{r}$, so $L+L_{r} \approx L$. From (9), there are

$$
v_{\text {in }}=(1-D) v_{c}, \quad I_{L}=\frac{v_{c}}{R(1-D)},
$$

where $D$ is the steady state switching duty cycle and $I_{L}$ is the steady state inductor current.

3.2. Negative Input Voltage. For negative input voltage, from Figure 4(a), when the switch $S$ is turned on, the state differential equations are

$$
\begin{gathered}
L \frac{\mathrm{d} i_{L}}{\mathrm{~d} t}=-v_{\text {in }}, \\
C_{o} \frac{\mathrm{d} v_{c}}{\mathrm{~d} t}=i_{L_{r}}-\frac{v_{c}}{R} .
\end{gathered}
$$


From Figure 4(b), when the switch $S$ is turned on, the corresponding state differential equations are

$$
\begin{gathered}
L \frac{\mathrm{d} i_{L}}{\mathrm{~d} t}=-v_{\mathrm{in}}, \\
C_{o} \frac{\mathrm{d} v_{c}}{\mathrm{~d} t}=-\frac{v_{c}}{R} .
\end{gathered}
$$

From Figure 4(c), when the switch $S$ is turned off, the corresponding state differential equations are

$$
\begin{aligned}
\left(L+L_{r}\right) \frac{\mathrm{d} i_{L}}{\mathrm{~d} t} & =-v_{\text {in }}-v_{c_{r}}, \\
C_{o} \frac{\mathrm{d} v_{c}}{\mathrm{~d} t} & =-\frac{v_{c}}{R} .
\end{aligned}
$$

According to the above analysis, it can be seen that the resonant capacitor voltage satisfies (4) when the switch $S$ is turned off. From Figure 5, at the end of switching cycle, the resonant capacitor voltage is equal to $\Delta v_{c_{r}}-v_{c}$; thus, the integration of the resonant capacitor voltage is $-v_{c}$ in the time.

The matrix representation of the state variables is $\mathbf{x}=$ $\left[\begin{array}{ll}i_{L} & v_{c}\end{array}\right]^{T}$; according (11), (12), and (13), the state space equation is

$$
\dot{\mathbf{x}}=A_{i} \mathbf{x}+B_{i} \mathbf{u}, \quad i=1,2,3,
$$

where

$$
\begin{array}{cc}
A_{1}=\left[\begin{array}{cc}
0 & 0 \\
0 & -\frac{1}{R C_{o}}
\end{array}\right], & B_{1}=\left[\begin{array}{c}
\frac{-v_{\text {in }}}{L} \\
i_{L_{r}} \\
C_{o}
\end{array}\right], \\
A_{2}=\left[\begin{array}{cc}
0 & 0 \\
0 & -\frac{1}{R C_{o}}
\end{array}\right], & B_{2}=\left[\begin{array}{c}
\frac{-v_{\text {in }}}{L} \\
0
\end{array}\right], \\
A_{3}=\left[\begin{array}{cc}
0 & 0 \\
0 & -\frac{1}{R C_{o}}
\end{array}\right], & B_{3}=\left[\begin{array}{c}
\frac{-v_{\text {in }}-v_{c_{r}}}{L+L_{r}} \\
0
\end{array}\right] .
\end{array}
$$

Therefore, for negative input voltage, the GSSA equation of the Bipolar-Gain Pseudo-Boost converter can be obtained as

$$
\frac{\mathrm{d}}{\mathrm{d} t}\left[\begin{array}{l}
i_{L} \\
v_{c}
\end{array}\right]=\left[\begin{array}{cc}
0 & 0 \\
0 & -\frac{1}{R C_{o}}
\end{array}\right]\left[\begin{array}{l}
i_{L} \\
v_{c}
\end{array}\right]+\left[\begin{array}{c}
\frac{-v_{\mathrm{in}}}{L}+\frac{v_{c}}{L}(1-d) \\
\frac{i_{L}(1-d)}{C_{o}}
\end{array}\right] .
$$

The equation $(\mathrm{d} / \mathrm{d} t)\left[\begin{array}{c}i_{L} \\ v_{c}\end{array}\right]=0$ is founded at steady state because $L \gg L_{r}$, so $L+L_{r} \approx L$. From (16), there are

$$
v_{\text {in }}=(1-D) v_{c}, \quad I_{L}=\frac{v_{c}}{R(1-D)} .
$$

According to (10) and (17), no matter if the input voltage is positive or negative, the voltage transfer ratios of BipolarGain Pseudo-Boost converter keep the same. The converter has the same Boost characteristic as the traditional Boost converter. In addition, the voltage transfer ratio does not rely on the resonant branch parameters and switching frequency, which is determined only by the switching duty cycle.

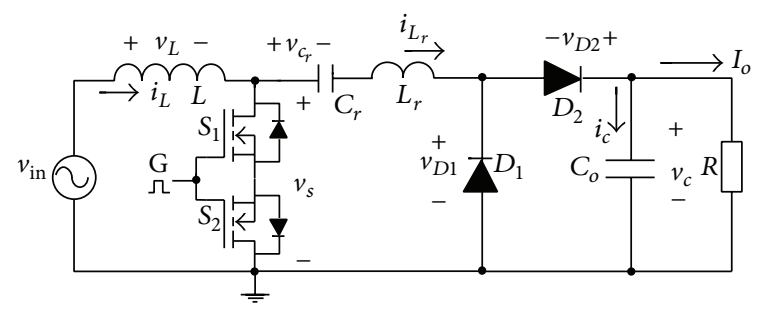

FIgURE 6: The topology of bridgeless Bipolar-Gain Pseudo-Boost PFC converter.

\section{The Implementation of Bridgeless Bipolar-Gain Pseudo-Boost Converter}

The voltage transfer ratio of traditional DC-DC converter is unipolar, which means that it only makes positive and negative input voltage transfer to positive output voltage. However, the PFC converter belongs to AC-DC converter, which makes AC input voltage transfer to DC output voltage. Hence, the traditional DC-DC converter cannot be used as PFC converter. To realize PFC, the most direct way is combining a front-end bridge rectifier with a DC-DC converter. Assume that the voltage transfer ratio of DC-DC converter is bipolar; that is, no matter if the input voltage is positive or negative, the output voltage is always positive. This DC-DC converter can realize AC-DC transformation, which eliminates the bridge of traditional AC-DC converter and improves the efficiency of the converter.

According to the steady state characteristic of BipolarGain Pseudo-Boost converter, it is shown that the converter has bipolar-gain characteristic. So the Bipolar-Gain PseudoBoost converter can be used as PFC converter. From Figure 6, the bridgeless PFC converter can be achieved by using bidirectional switch $S_{1}$ combined with $S_{2}$ instead of controllable switching $S$ of the Bipolar-Gain Pseudo-Boost converter. The converter has some advantages such as simple topology, high efficiency, and simple controlled circuit. Compared with the double bridge-leg bridgeless PFC converter researched in paper $[3,14-16]$, the converter has reduced the EMI due to common-grounded input and output. Compared with the bridgeless PFC converter proposed in paper [3, 13-17], the Bipolar-Gain Pseudo-Boost converter has improved the utilization ratio and reduced the costs.

From the preceding analysis, the achievement conditions of bridge Pseudo-Boost PFC converter are as follows.

(1) From Figures 3 and 5, a two-way switch is needed because the switch $S$ should suffer positive and negative voltage.

(2) The choice of inductor $L$ : to guarantee that the converter operates in CCM, the inductor $L$ must satisfy

$$
\frac{v_{\text {inp, } \max }}{L} D_{\max } T_{s} \leq 2 I_{\text {inp }}
$$

The input current effective value is $I_{\text {inp }}=\sqrt{2} P_{o} /$ $\eta v_{\mathrm{in}, \mathrm{RMS}}$, where $v_{\mathrm{in}, \mathrm{RMS}}$ is the input voltage effective 


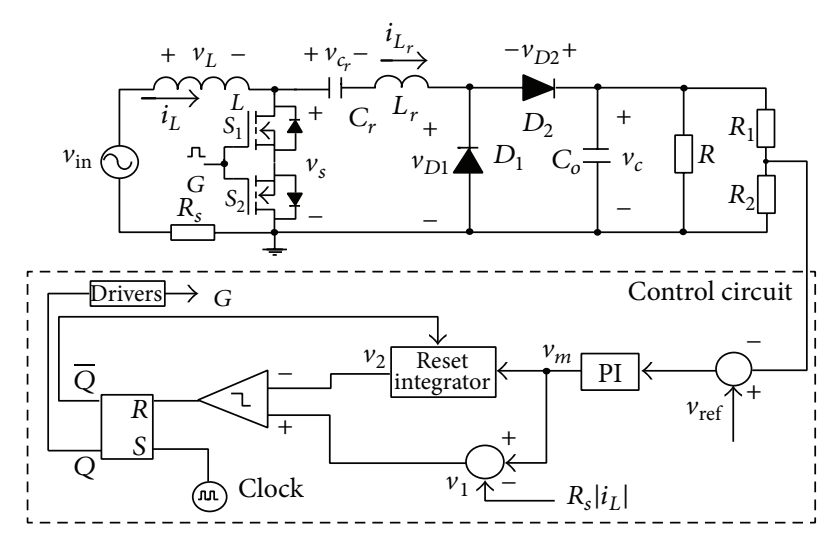

FIGURE 7: Block diagram of one-cycle control bridgeless BipolarGain Pseudo-Boost PFC converter.

value, $v_{\text {inp,max }}$ is the maximum input voltage peak value, $P_{o}$ is the output power, and $\eta$ is the efficiency of the converter. In addition, $D_{\max }$ is the maximum duty cycle and $T_{s}$ is the switching cycle.

(3) The choice of resonant branch parameters: from (2), (4), and the operation figure, it can be obtained as

$$
\Delta v_{c_{r}}=\frac{I_{L}}{2 C_{r}}\left(T_{s}-D T_{s}\right)=\frac{v_{c}}{2 R C_{r}} T_{s} .
$$

From (19), the choice of $\Delta v_{c_{r}}$ has nothing to do with resonant inductor $L_{r} . \Delta v_{c_{r}}$ is too large to increase the switch voltage stress, so larger resonant $C_{r}$ and smaller switching period $T_{s}$ should be chosen with the condition that resonant capacitor $C_{r}$ is much smaller than output capacitor $C_{o}$. However, the resonant capacitor $C_{r}$ is too small to result in zero-crossing distortion, and it must satisfy $v_{c}>\Delta v_{c_{r}}>0$; moreover,

$$
D_{\min } T_{s}>\frac{T_{r}}{2}
$$

where the minimum duty cycle is $D_{\min }=1-v_{\text {inp, max }} / v_{c}$, and the resonant period is $T_{r}=2 \pi \sqrt{L_{r} C_{r}}$. Thus, a suitable resonant inductor $L_{r}$ should be chosen with respect to (19) when the resonant inductor $L_{r}$ is much smaller than the Boost inductor $L$.

\section{One-Cycle Control Bridgeless Bipolar-Gain Pseudo-Boost Converter}

Figure 7 is the principle scheme of bridgeless PseudoBoost PFC converter based on one-cycle control technology. The main circuit topology employs a bidirectional switch composed of $S_{1}$ and $S_{2}$. Dotted part of Figure 7 is the onecycle control circuit, which consists of an integrator with reset function, a comparator, a RS trigger, and a clock signal generator.

The output voltage $v_{c}$ is sampled in each period and compared with a reference voltage $V_{\text {ref }}$. The compared result is compensated by PI regulator and then the modulation signal

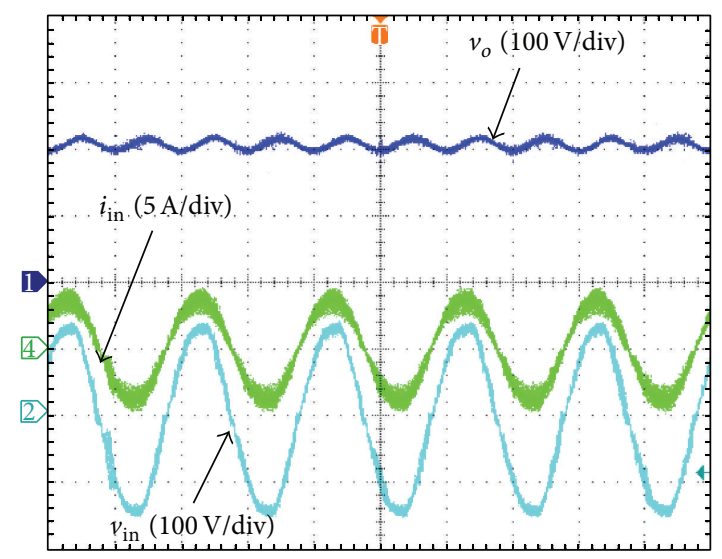

FIGURE 8: Waveforms of DC output voltage $v_{c}$, AC input voltage $v_{\text {in }}$, and $\mathrm{AC}$ input current $i_{L}$.

$v_{m}$ is obtained. At each clock pulse coming, the two-way switch is turned on. Meanwhile, the modulation signal $v_{m}$ begins to be integrated by the reset integrator. The difference values $v_{1}$ between $v_{m}$ and $R_{s}\left|i_{L}\right|$ are compared with the output signal $v_{2}$ from reset integrator, when $v_{2}=v_{1}$, and the output level of the comparator is flipped and the switch is turned off, while the reset integrator resets to zero until the next clock pulse arrives.

According to the principle of one-cycle control, it can be obtained that

$$
v_{m}-R_{s}\left|i_{L}\right|=\frac{1}{T_{s}} \int_{0}^{T_{s}} D v_{m} \mathrm{~d} t
$$

where $v_{m}=v_{c} R_{s} / R_{e}$ and $R_{e}$ is the equivalent input resistor of the bridgeless Bipolar-Gain Pseudo-Boost PFC converter and $R_{s}$ is the sampling resistor of inductor current.

When (21) is applied at each switching cycle, the output voltage of the bridgeless Bipolar-Gain Pseudo-Boost PFC converter is steady; moreover, the input current that is $i_{L}$ will maintain sinusoidal, and thus the purpose of PFC is achieved.

\section{Experiment Verification}

In order to verify the accuracy of the theoretical analysis, a set of experiments are designed. According to (18)-(20), the parameters of experiment circuit are as follows: $P_{o}=100 \mathrm{~W}$, $v_{\text {in, RMS }}=50 \mathrm{~V}, v_{c}=100 \mathrm{~V}, C=470 \mu \mathrm{F}, f_{\text {line }}=50 \mathrm{~Hz}, f=$ $50 \mathrm{kHz}, L=2.5 \mathrm{mH}, L_{r}=7.8 \mu \mathrm{H}$, and $C_{r}=330 \mathrm{nF}$.

Figure 8 shows the input voltage, input current, and output voltage waveforms of bridgeless Bipolar-Gain PseudoBoost PFC converter with $P_{o}=100 \mathrm{~W}$. It can be observed from Figure 8 that the output voltage of the converter $v_{c}$ is constant and the input current $i_{\text {in }}$ closely follows the input voltage $v_{\text {in }}$ to realize power factor correction.

Figure 9 shows the efficiency curve of bridgeless PseudoBoost PFC converter with $v_{c}=100 \mathrm{~V}$. It can be seen that the proposed converter has relatively high efficiency.

Figures 10(a) and 10(b) show the switch voltage, diode voltage, and inductor current waveforms for the positive 


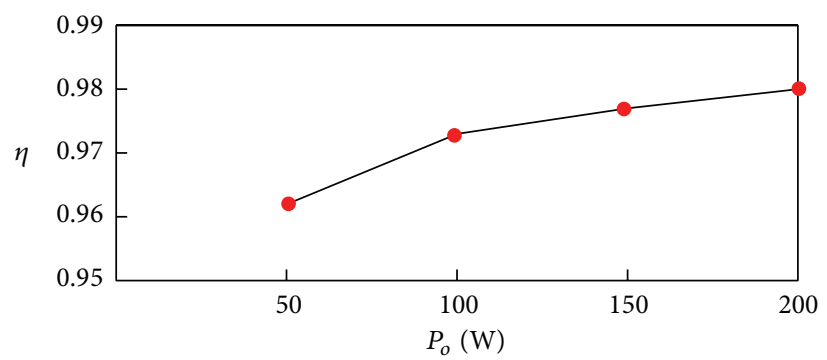

FIGURE 9: Efficiency of the converter with $100 \mathrm{~V}$ DC output voltage.

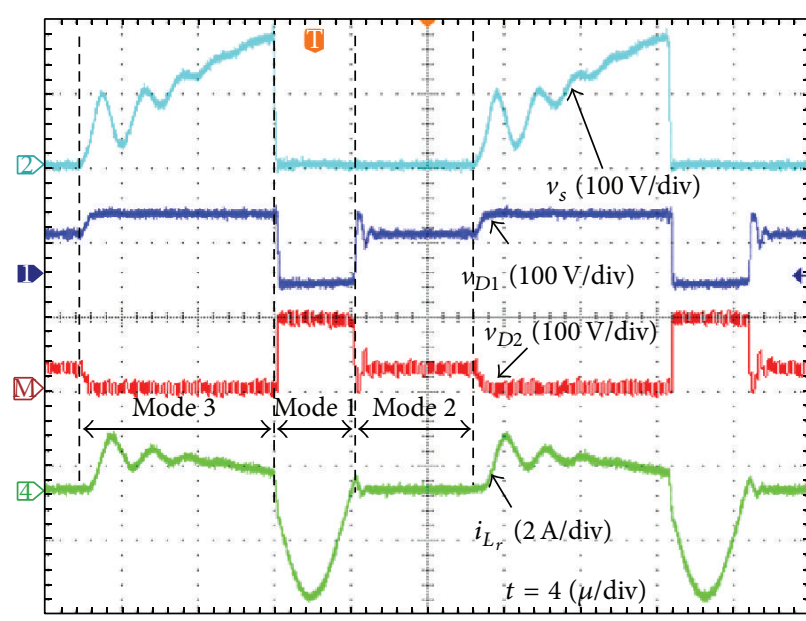

(a)

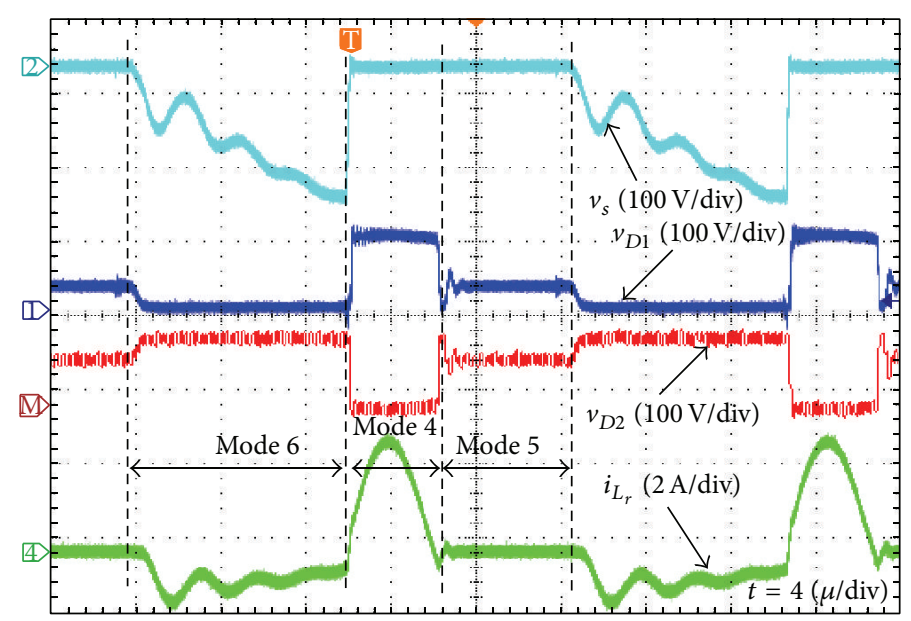

(b)

FIGURE 10: The key operation waveforms of the converter (a) with positive input voltage and (b) with negative input voltage.

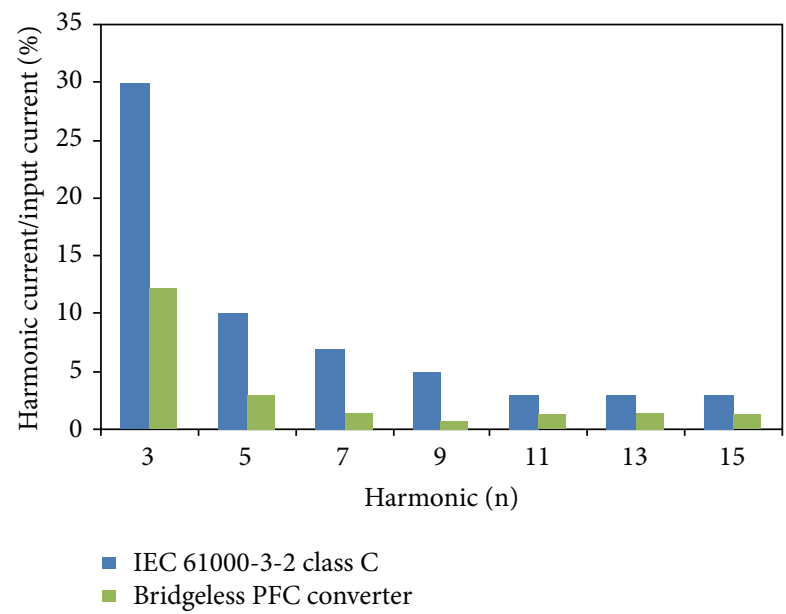

FIGURE 11: Measured harmonic contents of the proposed converter.

input voltage and negative voltage. It is seen that the experiment waveforms are consistent with the theoretical analysis waveforms and the diodes $D_{1}$ and $D_{2}$ can realize zero-current switching.

The measured harmonic contents of the line current at $100 \mathrm{~V}$ AC input voltage are shown in Figure 11 together with the limits of IEC61000-3-2 class D standard. It is shown that

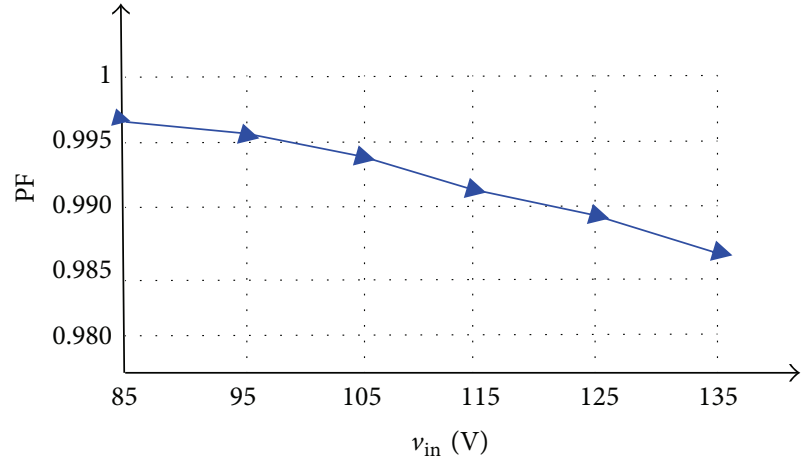

FIGURE 12: Measured PF value at full load of the proposed converter as the function of input voltage.

the input current harmonics of proposed converter can meet the IEC61000-3-2 standard. In addition, Figure 12 illustrates the measured PF value at full load of the proposed converter as the function of input voltage.

\section{Conclusion}

In this paper, we research the Bipolar-Gain Pseudo-Boost converter, and the topology and operation principles in 
CCM and DC steady-state character of this converter are analyzed. Based on this topology, a novel bridgeless BipolarGain Pseudo-Boost PFC converter is proposed. In addition, the implement condition of the bridgeless PFC and control technology of the converter is studied. The experiments show that the proposed converter is capable of achieving the PFC better, and zero-current switching of the fast recovery diode is achieved. Moreover, the reverse-recovery loss of the diode is reduced and the efficiency of the converter is improved.

\section{Conflict of Interests}

The authors declare that there is no conflict of interests regarding the publication of this paper.

\section{Acknowledgments}

This work was supported by the Applied Basic Research Programs of Technology Department under Foundation of Sichuan Province of China (no. 2012JY0120/12209596), Technology Support Programs of Technology Department of Sichuan Province (no. 2013GZ0130), the Educational Commission of the Sichuan Province of China (no. 11ZA003/11209435), the Key Programs of Xihua University (no. Z1120940), and Key Laboratory of University Project of Sichuan Provincial (application promotion and solar technology integration).

\section{References}

[1] Q. Chen, J.-P. Xu, Z. Y. Chen, and F. Zhang, "Research on bridgeless DCM pseudo boost PFC converter with high efficiency," Power Electronic Technology, vol. 46, no. 11, pp. 53-62, 2012.

[2] Q. Chen, J.-P. Xu, Z.-Y. Chen, and X.-S. Liu, "Research on bipolar gain Boost converter," Advanced Technology of Electrical Engineering and Energy, vol. 32, no. 4, pp. 44-48, 2013.

[3] A. J. Sabzali, E. H. Ismail, M. A. Al-Saffar, and A. A. Fardoun, "New bridgeless DCM sepic and Cuk PFC rectifiers with low conduction and switching losses," IEEE Transactions on Industry Applications, vol. 47, no. 2, pp. 873-881, 2011.

[4] W. Wang and D. D. C. Lu, "A bridgeless DIVM buck PFC rectifier with digital control and voltage doubler configuration," International Journal of Power Electronics, vol. 5, no. 2, pp. 125144, 2013.

[5] A. A. Fardoun, E. H. Ismail, A. J. Sabzali, and M. A. Al-Saffar, "New efficient bridgeless cuk rectifiers for PFC applications," IEEE Transactions on Power Electronics, vol. 27, no. 7, pp. 32923301, 2012.

[6] M. Mahdavi and H. Farzanehfard, "Bridgeless SEPIC PFC rectifier with reduced components and conduction losses," IEEE Transactions on Industrial Electronics, vol. 58, no. 9, pp. 41534160, 2011.

[7] Z. Chen, J. Xu, G. Zhou, and F. Zhang, "Analysis of bridgeless pseudo-boost PFC converter," in Proceedings of the 21st IEEE International Symposium on Industrial Electronics (ISIE '12), pp. 189-193, IEEE, Hangzhou, China, May 2012.

[8] T. Jiang, P. Mao, and S.-J. Xie, "Distortion issue on input current of OCC-PFC converter and its solution," Proceedings of the Chinese Society of Electrical Engineering, vol. 31, no. 12, pp. 51-56, 2011.
[9] B. Liu, J. J. Wu, J. Li, and J. Y. Dai, "A novel PFC controller and selective harmonics suppression," International Journal of Electrical Power \& Energy Systems, vol. 44, no. 1, pp. 680-687, 2013.

[10] D. Bortis, L. Fassler, and J. W. Kolar, "Comprehensive analysis and comparative evaluation of the isolated true bridgeless Cuk single-phase PFC rectifier system," in Proceedings of the IEEE 14th Workshop on Control and Modeling for Power Electronics (COMPEL '13), pp. 1-9, 2013.

[11] M. N. M. Hussain, M. F. M. Idris, I. Intan Rahayu et al., "Development of PI controller for battery charger using PFC rectifier," in Proceedings of the IEEE International Symposium on Power Electronics, Electrical Drives, Automation and Motion (SPEEDAM '10), pp. 1099-1101, June 2010.

[12] W. Wang and D. Dah-Chuan Lu, "A bridgeless DIVM buck PFC rectifier with digital control and voltage doubler configuration," International Journal of Power Electronics, vol. 5, no. 2, pp. 125144, 2013.

[13] D. Bortis, L. Fassler, and J. W. Kolar, "Comprehensive analysis and comparative evaluation of the isolated true bridgeless Cuk single-phase PFC rectifier system," in Proceedings of the IEEE 14th Workshop on Control and Modeling for Power Electronics (COMPEL '13), pp. 1-9, IEEE, Salt Lake City, Utah, USA, June 2013.

[14] Y. Chen, J. Zhou, W. P. Dai et al., "Application of improved bridgeless power factor correction based on one-cycle control in electric vehicle charging system," Electric Power Components and Systems, vol. 42, no. 2, pp. 112-123, 2014.

[15] K. H. Wu, J. H. Chiu, and Y. K. Lo, "A single-stage high powerfactor bridgeless AC-LED driver for lighting applications," International Journal of Circuit Theory and Applications, vol. 42, no. 1, pp. 96-109, 2014.

[16] A. Karaarslan and I. Iskender, "Analysis and comparison of current control methods on bridgeless converter to improve power quality," International Journal of Electrical Power and Energy Systems, vol. 51, pp. 1-13, 2013.

[17] E. H. Ismail, "Bridgeless SEPIC rectifier with unity power factor and reduced conduction losses," IEEE Transactions on Industrial Electronics, vol. 56, no. 4, pp. 1147-1157, 2009.

[18] Y. Jang and M. M. Jovanović, "Bridgeless high-power-factor buck converter," IEEE Transactions on Power Electronics, vol. 26, no. 2, pp. 602-611, 2011. 


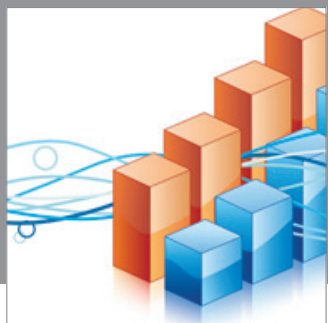

Advances in

Operations Research

mansans

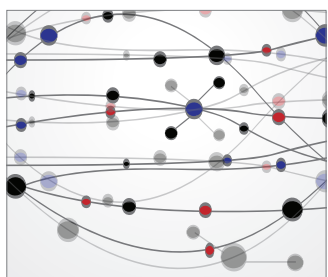

The Scientific World Journal
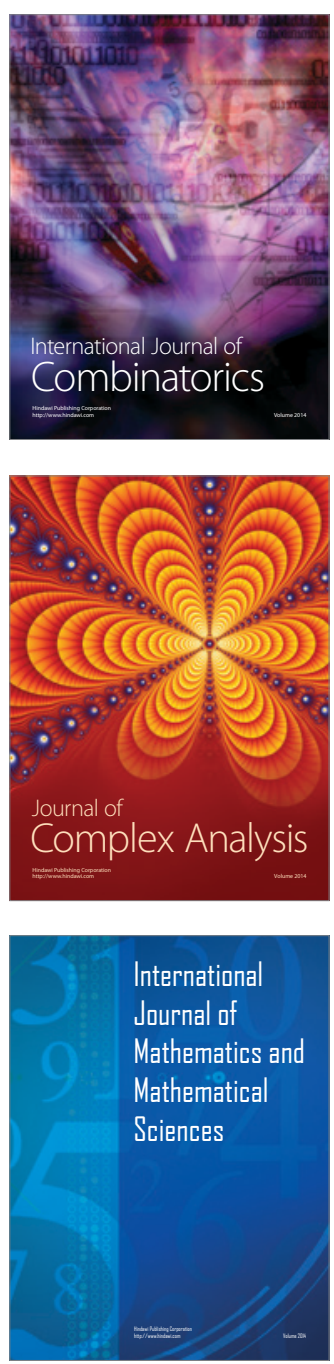
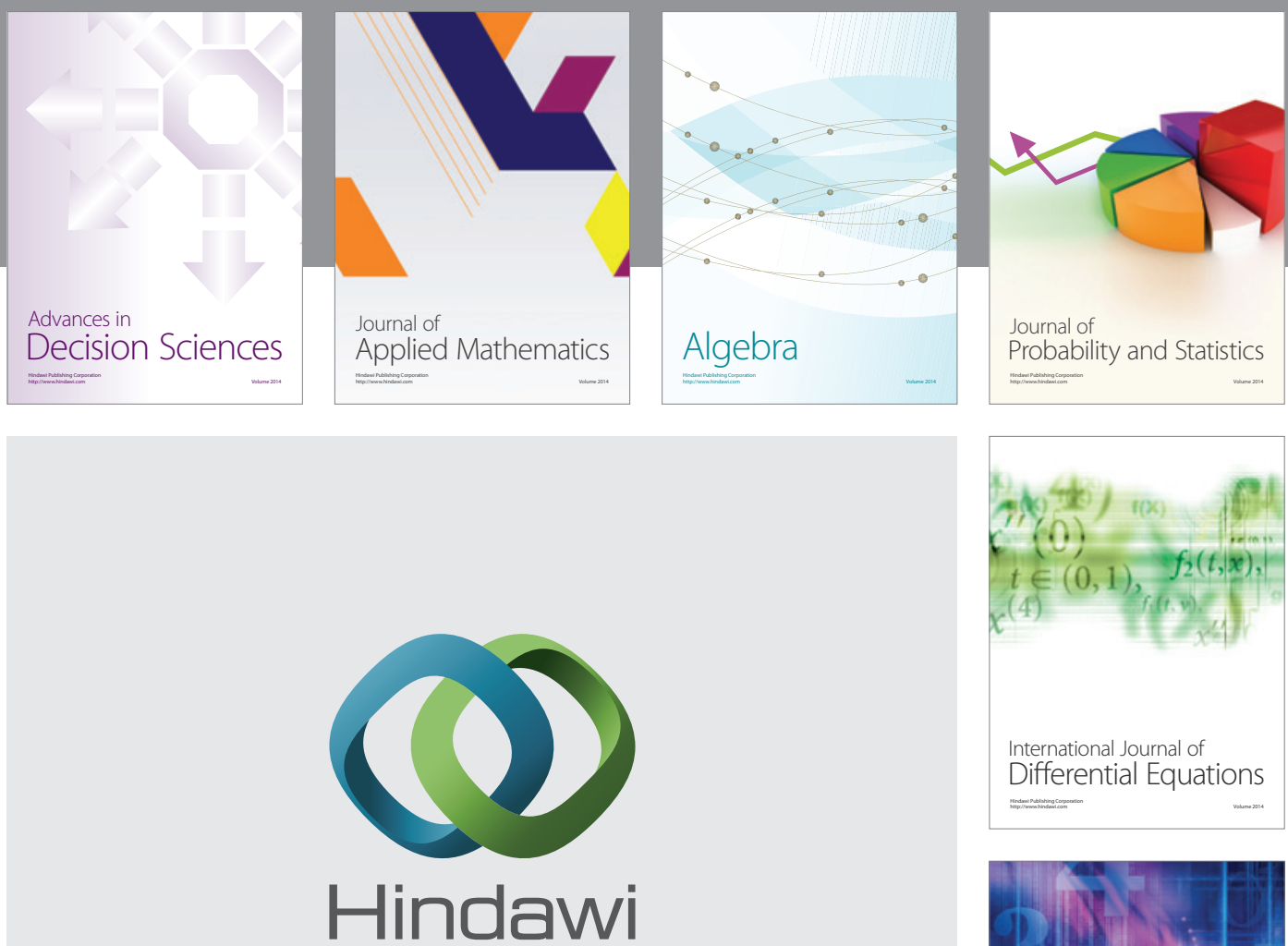

Submit your manuscripts at http://www.hindawi.com
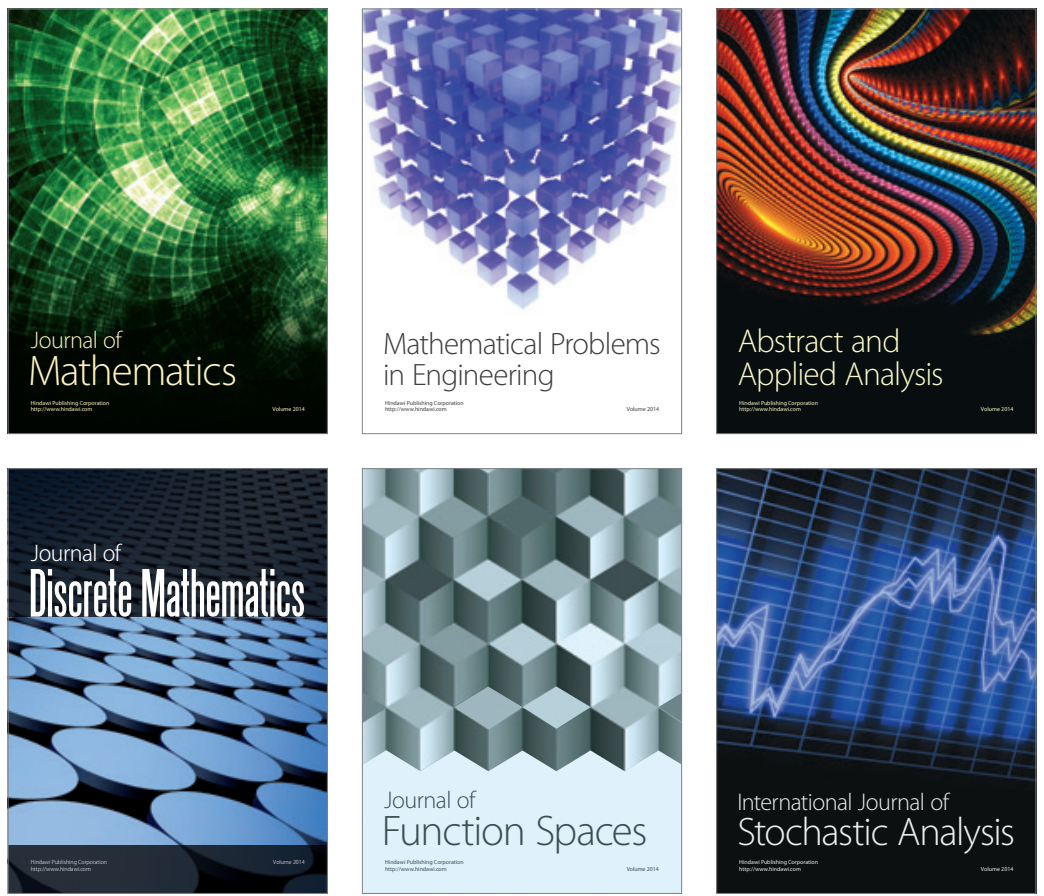

Journal of

Function Spaces

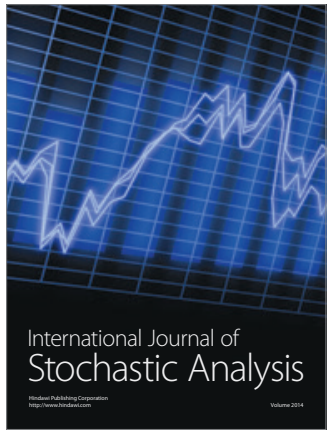

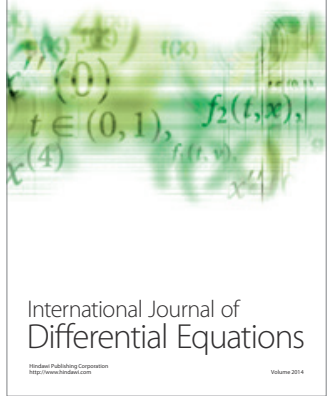
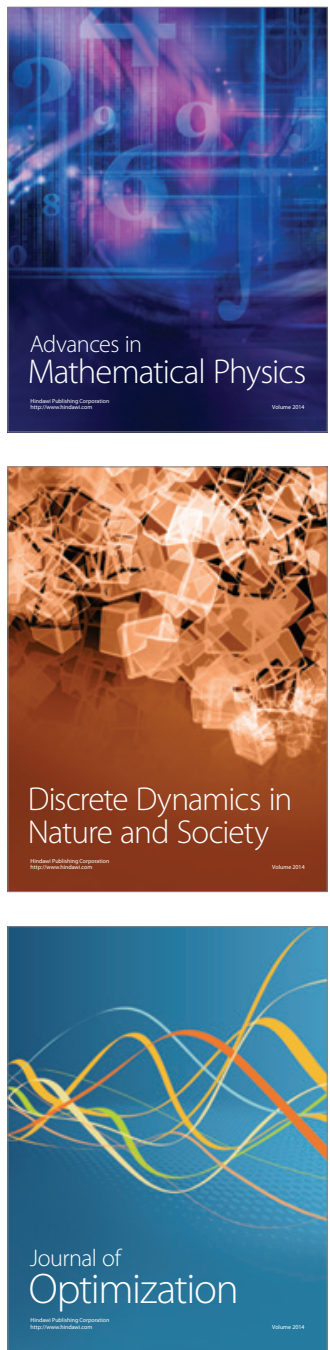